\title{
KONFLIKTE
}

\section{Beobachten und eingreifen}

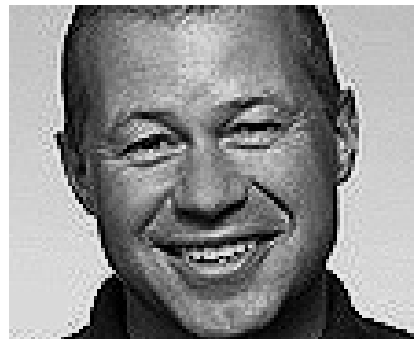

VON THOMAS FISCHER

Thomas Fischer leitet Konfliktmanagementseminare bei der K-Akademie der Unternehmensberatung Dr. Kraus \& Partner in Bruchsal.

Internet

http://www.k-akademie.de

\author{
Soll ein Vorgesetzter in einen Konflikt zwischen Mit- \\ arbeitenden eingreifen? Und wenn ja, wann und wie? \\ Vor diesen Fragen stehen Führungskräfte häufig, wenn \\ Mitarbeitende »nicht miteinander können«.
}

Konflikte unter Kollegen sollten stets gelöst werden, und zwar so, dass beide Seiten damit voll zufrieden sind. Das ist eine verbreitete Meinung. Doch Win-win-Lösungen sind oft nicht möglich. Dann gilt es, Kompromisse zu schließen oder zuweilen gar mit dem Konflikt zu leben. Wo Menschen zusammenarbeiten, gibt es schließlich unterschiedliche Meinungen und Interessen - und also auch Reibereien. Als Führungskraft sollten Sie daher wissen:

- Wann liegt überhaupt ein Konflikt vor? Und:

- Wann handelt es sich nur um einen Interessengegensatz oder eine Meinungsverschiedenheit?

Ein Beispiel: Sachbearbeiter Schaffer macht regelmäßig Überstunden, seine Kollegin Frohgemut geht stets Punkt 16 Uhr. Dies ist kein Konflikt, wenn beide dies Okay finden. Nehmen wir an, Herr Schaffer möchte auch früh gehen und Frau Frohgemut müsste dafür länger bleiben. Sie weigert sich aber »wegen der Kinder «. Auch das ist kein Konflikt, wenn Herr Schaffer dies akzeptiert. Dieser besteht erst, wenn Herr Schaffer denkt: »Immer soll ich auf diese Egoistin Rücksicht nehmen. « Denn nun behindern sich die Kollegen wechselseitig beim Erreichen ihrer Unternehmensziele und Herr Schaffer fühlt sich mit seinen Bedürfnissen nicht ernst genommen. Deshalb ist er verletzt.

Einen Konflikt kennzeichnen also drei Elemente:

- eine gegenseitige Zielbehinderung

- eine wechselseitige Abhängigkeit und
- eine Verletzung auf der Beziehungsebene.

Greifen Sie als Führungskraft nur ein, wenn ein Konflikt besteht - und zwar ein leistungsmindernder. Klären Sie dann zunächst, ob die Betroffenen sich des Konflikts bewusst sind. Und falls ja, ob Sie als Moderator erwünscht sind.

\section{Regeln für das Gespräch vereinbaren}

Werden Sie als Moderator akzeptiert, dann sorgen Sie dafür, dass Sie während des Gesprächs ungestört sind. Und bauen Sie gleich zu Beginn Vertrauen auf, indem Sie offen das Ziel des Gesprächs nennen nämlich die Voraussetzungen für eine effektive Zusammenarbeit wieder herzustellen. Definieren Sie anschließend Regeln für das Gespräch, beispielsweise dass jeder den anderen ausreden lässt.

Erst danach beginnt die Konfliktbearbeitung. Dazu sollte zunächst Kollege A schildern, wie er die Situation erlebt und Kollege B stellt Verständnisfragen. Dann tauschen A und B die Rollen. Um Missverständnisse zu vermeiden, sollte anschließend jeder Mitarbeiter in eigenen Worten beschreiben, wie sein Kollege den Konflikt sieht.

Anhand dieser unterschiedlichen Sichtweisen ermitteln sie gemeinsam die (realen) Ursachen des Konflikts. Erst danach können sie mit dessen Lösung beginnen. Hierbei hat sich folgendes Vorgehen bewährt. Bitten Sie die Mitarbeiter zunächst nur für sich selbst - zu formulieren: Welches Verhalten er sich vom Kol- 


\section{Welche Strategien für Konfliktgespräche denkbar sind}

\begin{tabular}{|c|c|c|}
\hline Strategie & Anwendung & Tipp \\
\hline Vermeiden & $\begin{array}{l}\text { Konfliktlösung ist nicht möglich } \\
\text { Konflikt besteht nur vorübergehend } \\
\text { den Konflikt anzusprechen schadet mehr als dessen } \\
\text { Lösung bringen würde }\end{array}$ & $\begin{array}{l}\text { Haben Sie sich dazu entschlossen, den Kon- } \\
\text { flikt zu vermeiden? Dann schließen Sie } \\
\text { auch mit ihm ab! }\end{array}$ \\
\hline Nachgeben & $\begin{array}{l}\text { taktisch nachgeben, um beim nächsten Mal etwas zu } \\
\text { erhalten } \\
\text { die Interessen der anderen Seite sind wichtiger als die } \\
\text { eigenen } \\
\text { wenn ein Durchsetzen unrealistisch wäre } \\
\text { wenn Sie im Verlauf des Konfliktes erkennen, dass der } \\
\text { andere Recht hat }\end{array}$ & $\begin{array}{l}\text { Falls Sie nachgeben wollen, tun Sie es } \\
\text { schnell und erläutern Sie Ihr Nachgeben } \\
\text { nachvollziehbar. Das entschärft die Situati- } \\
\text { on! }\end{array}$ \\
\hline Kompromiss finden & $\begin{array}{l}\text { die Interessen und die persönliche Beziehung beider } \\
\text { Parteien sind sehr wichtig } \\
\text { es ist zu aufwendig oder unmöglich, eine Lösung zu fin- } \\
\text { den, die beide vollkommen befriedigt } \\
\text { als vorübergehende Lösung }\end{array}$ & $\begin{array}{l}\text { Überlegen Sie vorher: Welche Punkte sind } \\
\text { für mich wichtig und für die Gegenparte } \\
\text { unwichtig? Und umgekehrt? So finden Sie } \\
\text { die Mitte. }\end{array}$ \\
\hline Sich durchsetzen & $\begin{array}{l}\text { die eigenen Ziele sind sehr wichtig } \\
\text { die persönliche Beziehung ist weniger wichtig } \\
\text { wenn keine andere Strategie möglich ist } \\
\text { bei Zeitdruck, in Notfällen }\end{array}$ & $\begin{array}{l}\text { Können Sie sich nicht durchsetzen, müssen } \\
\text { Sie den Konflikt eventuell durch Dritte lö- } \\
\text { sen lassen. Schalten Sie zum Beispiel Ihren } \\
\text { Vorgesetzten ein. }\end{array}$ \\
\hline Win-win-Lösungen finden & $\begin{array}{l}\text { die Interessen und die persönliche Beziehung beider } \\
\text { Parteien sind sehr wichtig } \\
\text { es ist genügend Zeit, um eine Lösung zu finden, die bei- } \\
\text { de Seiten vollkommen befriedigt }\end{array}$ & $\begin{array}{l}\text { Nutzen Sie Kreativitätstechniken wie } \\
\text { Brainstorming, um unterschiedliche Inter- } \\
\text { essen unter einen Hut zu bringen. }\end{array}$ \\
\hline
\end{tabular}

legen wünschen würde und was er selbst bereit wäre zu tun, damit der Konflikt gelöst wird. Danach beginnt das Aushandeln der Konfliktlösung. Als Moderator sollten Sie darauf achten, dass

- das Aushandeln der Lösung ein wechselseitiges Geben und Nehmen ist und

- keine Lösungen vereinbart werden, die zulasten Dritter oder der Organisation gehen.
Nach dem Aushandeln der Konfliktlösung sollten Sie schriftlich die Vereinbarungen und deren Umsetzung festhalten. Außerdem, was geschieht, wenn Vereinbarungen gebrochen werden sowie einen Folgetermin, um dies alles zu kontrollieren.

Noch ein Tipp: Das vorgestellte Vorgehen trägt dazu bei, dass das Gespräch sachlich verläuft. Trotzdem können die
Emotionen hochkochen. Versuchen Sie dann, das Gespräch so schnell wie möglich wieder auf die sachliche Ebene zurückzuführen. Sagen Sie zum Beispiel: »Ich sehe, wie stark der Konflikt Sie beide beschäftigt. Was halten Sie davon, wenn wir nun wieder zum vereinbarten Vorgehen zurückkehren, um eine Konfliktlösung zu erarbeiten. «Oder schlagen Sie eine kurze Pause vor.

\section{Wie man als Vorgesetzter seine Konfliktgespräche führt}

1. Bereiten Sie sich gut vor. Werden Sie sich über das Problem und Ihre Ziele klar.

2. Bleiben Sie gelassen. Versuchen Sie, vor dem Gespräch zu entspannen.

3. Sprechen Sie über Zahlen, Daten und Fakten. Bleiben Sie nicht allgemein, sondern bringen Sie konkrete Beispiele.

4. Sagen Sie Ich statt Wir: »Ich habe das Gefühl, dass ...", »Bei mir ist das so angekommen, dass..."

5. Der dosierte Einsatz von Emotionen ist in Ordnung. Aber bleiben Sie dabei ruhig: Wenn Sie die Kontrolle verlieren, schweben Sie in Gedanken in eine Ecke des Raums und betrachten Sie die Situation von außen. Oder machen Sie eine kurze Pause.

6. Formulieren Sie Kritik offen und ehrlich. Schockieren Sie dabei aber nicht.
7. Kritisieren Sie das Verhalten Ihres Gegenübers, nicht seine Person.

8. Suchen Sie gemeinsam nach Lösungen. Je mehr Lösungsvorschläge Sie besprechen, umso höher ist die Wahrscheinlichkeit, dass einer davon für beide geeignet ist.

9. Fassen Sie Zwischenergebnisse zusammen und fragen Sie nach, ob die Zusammenfassung richtig war. So beugen Sie Missverständnissen vor.

10. Sorgen Sie für konkrete Vereinbarungen. Halten Sie diese schriftlich fest und kontrollieren Sie diese Regeln.

11. Wenn die Situation eskaliert oder die Zeit knapp wird: Vertagen Sie das Gespräch.

12. Vereinbaren Sie einen Folgetermin zur Kontrolle, ob der Konflikt gelöst ist. 